\title{
Inquiétudes sur le devenir du Palais de la découverte
}

Les membres de la Société Française de Physique (SFP) sont depuis longtemps très attachés au Palais de la découverte, au sein du Grand Palais, au cœur de Paris. Le principe du Palais est, à son échelle, unique en France, par sa pédagogie active et originale. En dehors des expositions permanentes et temporaires, il propose chaque jour plusieurs dizaines d'exposés et d'ateliers permettant au public de découvrir, notamment à travers des expériences scientifiques réalisées en direct, des facettes de la science impossibles à présenter dans des expositions traditionnelles. Ces véritables shows de démonstration participative sont littéralement pris d'assaut par des classes venant de toute la France, et même de Belgique et de Suisse : la fréquentation annuelle du Palais de la découverte est de l'ordre de 600000 visiteurs, dont 25 à $30 \%$ de scolaires. Pendant 80 ans, il a marqué des générations et souvent déclenché un intérêt, voire une vocation, pour la démarche scientifique, notamment auprès des jeunes, en éveillant leur curiosité.

Le Palais de la découverte est né d'une initiative de Jean Perrin, lauréat en 1926 du prix Nobel de physique. En 1937, lors de l'exposition internationale « des Arts et des Techniques appliqués à la vie moderne », le Grand Palais est en grande partie investi par le Palais de la découverte. Fort du succès de ses plus de deux millions de visiteurs, il sera pérennisé, et rouvrira ses portes dès 1938. Sa surface sera peu à peu grignotée, notamment lors de la création des Galeries nationales, mais celle consacrée à la science et accessible au public atteint encore $11000 \mathrm{~m}^{2}$ à la fin des années 80, alors qu'elle n'est plus aujourd'hui que de $8000 \mathrm{~m}^{2}$. À chaque amputation, ce sont des thématiques qui disparaissent du Palais : c'est ainsi qu'on ne trouve plus par exemple de salle consacrée à l'énergie solaire ou aux semi-conducteurs.

Depuis 2010, le Palais de la découverte et la Cité des sciences et de l'industrie sont regroupés au sein d'Universcience, un établissement public à caractère industriel et commercial (EPIC), placé sous la double tutelle du ministère de l'Enseignement supérieur, de la Recherche et de I'Innovation et du ministère de la Culture. Le Palais de la découverte occupe toujours une partie du Grand Palais, géré maintenant par la Réunion des musées nationaux - Grand Palais, un autre EPIC créé en 2011.

Un gigantesque chantier de restauration du Grand Palais est en cours. II entrainera notamment la fermeture du Palais de la découverte de 2020 à 2024. À sa réouverture, un nouveau « Grand Palais des Arts et des Sciences » donnera accès, à partir d'un hall d'accueil unique situé au niveau de l'entrée actuelle des Galeries nationales, au Palais de la découverte et aux autres espaces du Grand Palais. Entre l'entrée et le Palais de la découverte proprement dit, un espace « Arts et Sciences » sera créé, pour assurer la continuité avec les Galeries nationales.

Malgré des perspectives a priori alléchantes, nous sommes très inquiets sur le devenir du Palais. La refonte complète des espaces entraine une nouvelle réduction d'un tiers de la surface « utile», qui sera à peine supérieure à $5000 \mathrm{~m}^{2}$. De plus, l'accent pourrait être mis sur la recherche scientifique contemporaine, au détriment des fondamentaux, en privilégiant des outils numériques.

La Société Française de Physique est convaincue que la vision de Jean Perrin - de proposer au public de « voir la science en train de se faire » à travers des démonstrations menées par des médiateurs qualifiés - est une des meilleures façons de présenter la démarche scientifique et est plus que jamais nécessaire aujourd'hui. La SFP suivra donc avec attention les prochaines étapes de ce chantier, et elle est prête à contribuer à la construction d'un projet avec tous ceux qui souhaitent que l'esprit du Palais perdure.

Daniel Hennequin

Michel Spiro

Président de la commission Culture scientifique de la SFP Président de la Société Française de Physique

\footnotetext{
Pour en savoir plus

- Le Palais de la découverte à l'exposition de 1937 : http://paris-projet-vandalisme.blogspot.fr/2016/10/le-palis-de-la-decouverte-lexposition.html - Une vidéo sur le projet de restauration : www.grandpalais.fr/fr/article/le-projet-de-restauration-et-damenagement-du-grand-palais

- Le numéro 410 (mai-juin 2017) de la revue Découverte, consacré à l'anniversaire des quatre-vingts ans du Palais.
} 